\title{
Delivery of Contracted Energy Flexibility from Communities
}

\author{
Rami El Geneidy ${ }^{1}$, Bianca Howard ${ }^{1}$ \\ ${ }^{1}$ Building Energy Research Group, School of Architecture, Building and Civil Engineering, \\ Loughborough University, United Kingdom
}

\begin{abstract}
This paper contributes to existing research on energy flexibility by introducing centralised and decentralised model-predictive control (MPC) strategies designed to deliver demand reductions over specific time periods for demand response based on real-time demand projections. The MPC strategies were evaluated with co-simulations of an English community fitted with heat pumps over three afternoons in January, March and November with a demand response event. Effects of dynamic and static electricity pricing on delivery of DR were analysed. In line with previous findings factors like seasonality and pricing were found to influence the flexibility potential of the community. Operational differences found between the centralised and decentralised MPC set-ups highlight importance of the control set-up for scalability and delivery of flexibility.
\end{abstract}

\section{Introduction}

Electrification of heating with heat pumps supported by the increasing amount of renewable electricity has been proposed to have good potential to reduce carbon emissions from heating (CCC, 2013). In the UK, this would allow shifting away from natural gas and oil, the current predominant domestic heating fuels (BEIS, 2018). But this is not unproblematic, because intermittency of renewable energy production and increased peak and base-load demand of electricity due to heating cause together significant challenges for balancing, required to maintain electricity grids stable (Jensen et al., 2017). This has increased the recognition of energy flexibility, which on the demand-side can be defined as the capability to cope with changes in energy supply through modifying demand (Jensen et al., 2017).

Residential buildings together with their heating, ventilation and cooling (HVAC) systems provide an inherent source of flexibility with the capability to store energy into the building fabric, also referred to as the 'thermal flywheel' (Haghighi, 2013). In electricity markets flexibility is typically realised through demand response (DR) schemes where a contractual relationship between parties sets requirements for de- livery of energy flexibility (U S Department of Energy, 2006). The term contracted flexibility is used in this paper used to describe this particular type of flexibility.

Contracted flexibility potential in electrically heated residential buildings could be significant. $28 \%$ of energy demand within the UK was accounted for domestic demand in 2017, of large portion for space heating (BEIS, 2018). To allow granular, decentralised actors like domestic buildings to offer contracted flexibility, the role of aggregators, parties managing them as assets and aggregating their contributions for electricity markets, has been established (Jensen et al., 2017). DR and balancing markets are gradually being opened to allow market entry for aggregators managing large volumes of demand-side resources.

For aggregators to use building HVAC systems for $\mathrm{DR}$, means to modify demand over set periods of time are required. To achieve this, model-predictive control (MPC) is attractive. In MPC, decisions are determined by solving an optimisation problem over a time horizon using a model of the building thermal response and external data inputs like weather forecasts and indoor temperature measurements (Killian and Kozek, 2016). For aggregators, most obvious approach to deliver DR would be centralised MPC structures where an aggregator would control the entire community and its systems with capability to set community-wide targets. This comes with drawbacks due to need for the buildings to share their data and centralised computation (Reynolds et al., 2017; Dusparic et al., 2017). Decentralised approaches require less sharing and communication of data with a centralised entity but could reduce optimality of the overall system (Reynolds et al., 2017; Dusparic et al., 2017; Mirakhorli and Dong, 2018).

Overall, research on MPC design for energy flexibility has been found to be limited (Clauß et al., 2017; Reynolds et al., 2017). Clauß et al. (2017) found in a review that only a few studies have introduced MPC strategies for buildings that explicitly considered flexibility objectives. A number of studies have investigated shaping energy demand either with price-based approaches through design of pricing sig- 
nals or incentive-based schemes to follow a reference demand (El Geneidy and Howard, 2018; Mirakhorli and Dong, 2018). For example, Cole et al. (2014) investigated how pricing signals could be used to reduce peak demand of a community with MPC aiming to minimise overall costs. Corbin and Henze (2017) presented a MPC strategy for DR to follow a reference demand profile derived from availability of renewable power generation. But to the authors' best knowledge, the capability of a community to follow a baseline for incentives while subjected to varying pricing schemes is yet to be analysed (El Geneidy and Howard, 2018).

This paper contributes to gaps in research by developing MPC strategies to deliver fixed demand reductions from a community, and evaluating its operation under static and dynamic pricing. The two MPC strategies, centralised and decentralised, are evaluated with co-simulations of MPC operation and community response. Simulations are performed over three afternoons in winter, spring and autumn to evaluate effects of seasons on the flexibility. Outcomes from the work contribute towards laying a foundation for identification of community energy flexibility potential and design of scalable strategies for aggregators to unlock it.

\section{Methods}

\section{Decentralised Control Strategy}

The decentralised control problem solved by each individual controller is presented in equations 1-5. The basic principle of the proposed MPC strategies is to use projections of future demand calculated by the MPC controller to define reference demand profiles for DR. Buildings in the community are incentivised to operate under this reference profile and penalised for exceeding it when DR is needed. Equations are written in their continuous form as interpreted by the simulation and optimisation tool used, JModelica.org (Åkesson et al., 2010). The software transcribes continuous equations written in Modelica into a general discrete form for numerical solving with the solver IPOPT.

The objective function is framed as an economic cost function which is minimised over time horizon $\left[t_{0}, t_{f}\right]$. $\gamma$ is the heat input which is being controlled and $p$ the electricity price. gamma g $_{\text {ref }}$ is a reference profile and $q$ the incentive to operate below this reference profile, and penalty for exceeding it. $T_{1}$ is the building internal temperature which by equation 4 is constrained to stay within certain time varying bounds signified by $T_{\min }(t)$ and $T_{\max }(t)$. To avoid infeasibilities but penalise for constraint violations, the slack variable $\epsilon$ is included along with a penalty for violations $r$.

$$
\begin{gathered}
\text { min. Cost }=\int_{t_{0}}^{t_{f}}(\gamma(t) p(t)+ \\
\left.\left(\gamma(t)-\gamma_{\text {ref }}(t)\right) q(t)+\epsilon r\right) d t \\
\frac{d T_{1}}{d t}=\frac{T_{1}-T_{2}}{R_{1} C_{1}}+\frac{A_{w i n} q_{h o r}}{C_{1}}+\frac{\gamma \eta Q_{c}}{C_{1}} \\
\frac{d T_{2}}{d t}=\frac{T_{o}-T_{2}}{R_{2} C_{2}}+\frac{T_{2}-T_{1}}{R_{1} C_{2}} \\
T_{\min }(t)-\epsilon \leq T_{1}(t) \leq T_{\max }(t)+\epsilon \\
0 \leq \gamma(t) \leq 1
\end{gathered}
$$

Equations 2 and 3 represent a simple grey-box thermal response model of the building. Connected resistance-capacitance (RC) elements has been a common method for characterising building thermal response for control purposes (De Coninck et al., 2016). $T_{1}, T_{2}$ and $T_{o}$ are temperatures of nodes representing indoor air, construction elements and outside, respectively. $R$ and $C$ are the thermal resistances and capacitances of each element. $q_{h o r}$ is the global horizontal irradiation. Each building is heated by a heat pump and $\gamma$ is the part load ratio of the heat pump compressor, $\eta$ the coefficient of performance (COP) and $Q_{c}$ the compressor power capacity. $A_{\text {win }}$ represents a coefficient which is used together with global horizontal irradiation to account for radiative gains through construction elements like windows. $\frac{d T_{1}}{d t}$ and $\frac{d T_{2}}{d t}$ are rates of change in temperatures of RC-elements over time.

An aggregator is allowed to make calls for DR by modifying $q(t)$ to set the incentive to follow a reference profile $\gamma_{r e f}$ over the DR period. To define $\gamma_{r e f}$, a 'load-shaping' heuristic, described by equation 6 , is used where the MPC projection from one time step prior to the DR call $\left(\gamma_{k-1}\right)$ is modified with a fixed demand reduction $\left(\gamma_{r}\right)$ over the DR period $\left(t_{D R}\right)$.

$$
\begin{aligned}
& \gamma_{r e f}=\gamma_{k-1}\left(t_{D R}\right)-\gamma_{r}\left(t_{D R}\right), \\
& \gamma_{r e f}=0 \text { if } \gamma_{k-1}\left(t_{D R}\right)-\gamma_{r}\left(t_{D R}\right) \leq 0
\end{aligned}
$$

\section{Centralised Control Strategy}

The centralised MPC strategy is presented by equations 7-12. The only difference to the decentralised strategy is that instead of solving separate problems for each building, optimisation is performed only once over the community consisting of $M$ buildings. In this case the aggregator would need full knowledge of the underlying system characteristics, their states and heating systems to perform the control. The load-shaping is performed similar to the decentralised strategy except that $\gamma_{r e f, i}$ is defined based on a community-level target where the overall demand of the community is reduced by a set amount and the reductions of individual buildings are left open to be solved through the centralised optimisation. 


$$
\begin{gathered}
\text { min. Cost }=\int_{t_{0}}^{t_{f}} \sum_{i=0}^{M}\left(\gamma_{i}(t) p(t)+\right. \\
\left.\left(\gamma_{i}(t)-\gamma_{\text {ref }, i}(t)\right) q(t)+\epsilon_{i} r\right) d t \\
\frac{d T_{1, i}}{d t}=\frac{T_{1, i}-T_{2, i}}{R_{1, i} C_{1, i}}+\frac{A_{i, w i n} q_{i, h o r}}{C_{1, i}}+\frac{\gamma \eta Q_{i, c}}{C_{1, i}},, \forall i \in M \\
\frac{d T_{2, i}}{d t}=\frac{T_{o}-T_{2, i}}{R_{2, i} C_{2, i}}+\frac{T_{2, i}-T_{1, i}}{R_{1, i} C_{2, i}}, \forall i \in M \\
T_{\min , i}(t)-\epsilon_{i} \leq T_{1, i} \leq T_{\max , i}(t)+\epsilon_{i},, \forall i \in M \\
0 \leq \gamma_{i}(t) \leq 1, \forall i \in M \\
i=[1,2, \ldots, M-1, M]
\end{gathered}
$$

The solutions given by both the centralised and decentralised MPC should be similar with the exception that the centralised solution should allocate demand reductions in an optimum manner to each building. For example, a building with more flexibility would also be asked to deliver more. However, the size of the problem, i.e. the amount of variables included in a single optimisation is significantly larger in the centralised strategy compared to the individual MPC problems solved in the decentralised strategy.

\section{Community Characteristics and Weather}

TEASER (Tool for Energy Analysis and Simulation for Efficient Retrofit) was used to create a set of building models written in Modelica to emulate a community for the simulation case studies with JModelica.org (Remmen et al., 2018). The models used to represent the buildings created with TEASER were single-zone third-order RC-models from the IBPSA model library for which parameters were calculated with TEASER (Remmen et al., 2018). These RCmodels have been validated according to the VDI 6007 Part 1 standard (Wetter and Van Treeck, 2017).

The case community consisted of six semi-detached and four terraced houses to approximately correspond with their respective portions in the UK housing stock (HM Government, 2016). The house and building material characteristics were based on standardised UK archetypes by Allen and Pinney (1990). House characteristics like geometry and orientation were varied within the community. All homes had occupancy starting at 17:00 in the afternoon and demand temperatures of 19 or $20{ }^{\circ} \mathrm{C}$. Table 1 summarises the geometries of the homes. Houses 1-6 were semidetached and 7-10 terraced. For further details about the approach used in the community model creation the reader can refer to El Geneidy (2018).

To model operation of the heating systems, the zone

\begin{tabular}{|c|c|c|c|c|c|c|c|}
\hline \multirow{2}{*}{\multicolumn{2}{|c|}{$\begin{array}{l}\text { House Total } \\
\text { nr. }\end{array}$}} & \multicolumn{3}{|c|}{ Element Area $\left[m^{2}\right]$} & \multirow[b]{2}{*}{$\begin{array}{l}\text { Inner } \\
\text { Wall+ } \\
\text { Roof }\end{array}$} & \multirow[b]{2}{*}{$\begin{array}{l}\text { Vol- } \\
\text { ume } \\
{\left[\mathrm{m}^{3}\right]}\end{array}$} & \multirow[b]{2}{*}{$\begin{array}{l}\text { Orien- } \\
\text { tation } \\
{\left[^{\circ}\right]}\end{array}$} \\
\hline & & $\begin{array}{l}\text { Grounc } \\
\text { Floor }\end{array}$ & $\begin{array}{l}\text { dWin- } \\
\text { dow }\end{array}$ & $\begin{array}{l}\text { Outer } \\
\text { Wall+ } \\
\text { Roof }\end{array}$ & & & \\
\hline \pm & 83.2 & 43.6 & 10.5 & 138 & 125.3 & 195.5 & 193.2 \\
\hline 2 & 87.4 & 41.5 & 10 & 141.6 & 126.1 & 205.5 & 256.1 \\
\hline 3 & 92 & 44.6 & 9.9 & 145 & 131.4 & 216.1 & 249.8 \\
\hline 4 & 86.4 & 38.8 & 10.6 & 141.9 & 124.7 & 203.1 & 255.8 \\
\hline 5 & 83.1 & 41.8 & 10.1 & 147 & 125.3 & 195.2 & 329 \\
\hline 6 & 93.5 & 43.7 & 10 & 147 & 134.3 & 219.7 & 206.9 \\
\hline 7 & 82.8 & 41.4 & 10.3 & 93.7 & 189.3 & 190.3 & 324.6 \\
\hline 8 & 82.4 & 41.2 & 10.5 & 85.6 & 186.6 & 189.6 & 200.7 \\
\hline 9 & 78.3 & 39.1 & 9.7 & 94.4 & 178.4 & 180 & 295.5 \\
\hline 10 & 79.4 & 39.7 & 9.7 & 89 & 174.5 & 182.5 & 282.2 \\
\hline
\end{tabular}
models were included with air-to-water heat pump and radiator models from the IBPSA model library. The radiator was discretised as a single element with a nominal inlet temperature of $55{ }^{\circ} \mathrm{C}$ and outlet of
Table 1: Geometries of the houses included in the case community for simulations.

$45{ }^{\circ} \mathrm{C}$. A constant mass-flow was maintained in the system with a pump, the consumption of this pump was omitted from the observed power demand. The heat pump was connected to a boundary of outside air and had a nominal temperature change of $10{ }^{\circ} \mathrm{C}$ in both the evaporator and condenser. A first-order polynomial efficiency curve of the form $C O P=C O P_{n o m} \times\left(a_{0}+a_{1} \gamma_{p l}\right)$ was used to account for changes in COP of the heat pump under part-load operation. 0.8 and 0.2 were as coefficients $a_{0}$ and $a_{1}$, respectively. Nominal COP $\left(C O P_{\text {nom }}\right)$ was set to 2.5 and compressor power capacity to $3 \mathrm{~kW}$. The Modelica code of the building models and the Python scripts used in the simulations have been made publicly available in Github (El Geneidy, 2019).

To observe effects of seasonality on MPC operation and flexibility, three different afternoons from winter, spring and autumn were chosen based on analysing the weather file, a CIBSE typical reference year (TRY) for Nottingham, UK. The case days chosen were: $7^{\text {th }}$ of January, $1^{\text {st }}$ of March and $21^{\text {st }}$ of November. In the afternoons the temperature and solar irradiation reduce as time passes during all days. Days in March and November have similar temperature profiles but in March the solar irradiation is higher. January has the coldest average temperature and lowest solar irradiation of the days. Afternoons were chosen because peaks in electricity prices occur typically in the early evenings in the UK, presenting best opportunities to gain benefits from demand reductions.

\section{Simulation Set Up}

To find parameters for the RC-models, a system identification was performed using MPCPy, Python package developed for MPC design (Blum and Wetter, 2017). Model parameters were defined for each case day by using parameter fitting in combination with Unscented Kalman Filter (UKF). First optimisation with JModelica.org was used to fit model parameters over a training period of two days. Before each sim- 
Table 2: Main input parameters used in the simulation case studies for reference and cases with DR calls.

\begin{tabular}{lc}
\hline Input Parameter & Value \\
\hline RBC PI proportional gain & 0.30 \\
RBC PI integral time constant & 1800 \\
RBC PI hysteresis term & \pm 0.2 \\
MPC time step $t_{k+1}-t_{k}[\mathrm{~min}]$ & 5 \\
Optimisation horizon length, $H[\mathrm{~h}]$ & 2 \\
Flexibility Cost, $q[£ / \mathrm{MWh}]$ & 150 \\
Constraint violation cost, $r$ & 1000 \\
Static Electricity Price, $p[£ / \mathrm{MWh}]$ & 50 \\
Demand Response call time & $17: 00$ \\
Demand Response start time & $17: 30$ \\
Demand Response end time & $18: 30$ \\
Requested total demand reduction $[\mathrm{kW}]$ & 2.0 \\
\hline
\end{tabular}

ulation UKF was used to train the model further for the case day with data spanning three hours, 13:0016:00 with a time step of ten minutes. Over the simulation period UKF was run every twenty minutes to continuously update the models.

JModelica.org was used to transcribe the optimisation problems for the solver, IPOPT which is designed to find local optimums for non-linear problems (Wächter and Biegler, 2005). The co-simulations were conducted in a step-wise manner where each step consisted of following phases: 1) the control problem was initialised with current internal temperatures of the house or houses, 2) the optimisation problem was solved over the control horizon and 3) the response of each house to the optimised control sequences was emulated. The time period simulated over each afternoon was 16:30-19:00. DR was implemented by a call to reduce demand $17: 30-18: 30$ by $2 \mathrm{~kW}$ with a call initiated at 17:00.

In total six simulations with DR were performed, one for each case day under static and dynamic electricity pricing. For comparisons with the MPC strategies two reference cases were run: rule-based control (RBC) and decentralised energy minimisation MPC. In RBC a PI-controller (Proportional Integrative) with hysteresis was set to follow the demand temperature of the homes. In the energy minimisation MPC cases an objective function to minimise cost with a static price signal was used.

Table 2 summarises the most important simulation inputs used in the co-simulations. Electricity pricing was set to reflect fluctuations typical in the UK wholesale market (Ofgem, 2018). 17:00-19:00 the price was set to vary between 81 and $99 £ /$ MWh and 0 and $40 £ / \mathrm{MWh}$ otherwise. When static pricing was considered, electricity price was $50 £ / \mathrm{MWh}$, approximately the long-term average wholesale electricity price in the UK (Ofgem, 2018).

For January the simulations were initialised by emu- lating the buildings without heat input at 16:00 with starting from indoor temperature of $20^{\circ} \mathrm{C}$. A similar initialisation process, starting at 12:00 was done for days in March and November. This way diversity among the starting temperatures between the houses while maintaining comparability between simulation cases was achieved. Collocation points were adjusted in JModelica.org to produce a constant heat input for each time step. For the first optimisation time step a randomised control profile was defined by setting $0.2 \leq \gamma \leq 0.6$ during occupied hours for each home. The same initial guess for demand was used in the decentralised and centralised cases. Weather predictions were assumed to be perfect in all simulation cases.

The MPC time step and time horizon were chosen to find a compromise between computational efficiency and system dynamics. It was necessary to cover the anticipated DR period of one hour and have a time step representing the underlying system dynamics. A very small time step would affect computational feasibility of the MPC problem because reducing the time step while maintaining the time horizon would mean increase in time steps as well as the simulation time steps. Also, heat pumps have been found to operate sub-optimally if cycling is very frequent(Technology, 2012).

The amount of variables in the optimisation problem interpreted by JModelica.org increased from 619 of the decentralised MPC problem to 5108 in the centralised MPC, which in initial simulations was found to significantly affect the capability to find optimum solutions in centralised MPC. It was hypothesised that the difference in amount of optimisation variables made the centralised MPC less "sensitive" to finding satisfactory optimum from the restoration phase, especially in the case for Spring when heat demand was low. To remedy this, the objective function was scaled with a factor of 100 in the centralised MPC cases. Overall, this demonstrates the difficulties associated with using non-linear optimisation solver like IPOPT compared to a linear optimisation solver where, if the problem is well-defined, finding a feasible optimum solution is well-guaranteed.

\section{Results}

\section{Demand Response Cases}

In table 3 results for energy consumption, total comfort violations and peak power are presented for all simulation cases. Overall, the RBC caused highest energy consumptions and comfort violations. However, for energy consumption this was partly due to the demand temperature being used as the set point instead of the lower temperature bound, which the energy minimisation MPC would follow. To improve performance of the $\mathrm{RBC}$, a more careful tuning of the PI-controller parameters would be required but 
this was left outside the scope of this study. Using MPC reduced comfort violations by preheating the houses prior to expected occupancy at 17:00, unlike the $\mathrm{RBC}$ which reacted to the change in programmed set-point causing reduction in the service-level. For the case day in March, some of the buildings warmed up during the day which increased the indoor temperature and caused comfort violations to occur even when heating demand was very low.

In all cases DR events increased energy consumption compared to energy minimisation. For example, the energy consumption on the winter case day was increased by $12-19 \%$. Overall, the centralised and decentralised MPC produced similar results in terms of overall energy consumption and comfort violations. Dynamic pricing increased energy consumption compared to static pricing. As an example, in January the increase in consumption due to dynamic pricing was around $6.0 \%$ with the decentralised MPC.

Table 3: Simulation case study results for energy consumption, comfort and peak power.

\begin{tabular}{|c|c|c|c|}
\hline Case & $\begin{array}{l}\text { Consum- } \\
\text { ption } \\
{[\mathrm{kWh}]}\end{array}$ & $\begin{array}{l}\text { Comfort } \\
\text { Violation } \\
{[\mathrm{Ch}]}\end{array}$ & $\begin{array}{l}\text { Peak } \\
\text { Power } \\
{[\mathrm{kW}]}\end{array}$ \\
\hline \multicolumn{4}{|c|}{ Winter - January } \\
\hline \multicolumn{4}{|l|}{ References } \\
\hline $\mathrm{RBC}$ & 35.26 & 2.88 & 20.11 \\
\hline Energy Min. & 20.88 & 0.53 & 17.85 \\
\hline \multicolumn{4}{|c|}{ DR - Static Pricing } \\
\hline Centralised & 23.34 & 0.31 & 20.66 \\
\hline Decentralised & 23.59 & 0.50 & 23.96 \\
\hline \multicolumn{4}{|c|}{ Dynamic Pricing } \\
\hline Centralised & 24.75 & 0.34 & 25.38 \\
\hline Decentralised & 24.86 & 0.42 & 25.44 \\
\hline \multicolumn{4}{|c|}{ Spring - March } \\
\hline \multicolumn{4}{|l|}{ References } \\
\hline $\mathrm{RBC}$ & 1.61 & 3.41 & 1.27 \\
\hline Energy Min. & 0.32 & 2.23 & 0.53 \\
\hline \multicolumn{4}{|c|}{ DR - Static Pricing } \\
\hline Centralised & 1.06 & 2.21 & 3.25 \\
\hline Decentralised & 0.67 & 2.18 & 2.57 \\
\hline \multicolumn{4}{|c|}{ DR - Dynamic Pricing } \\
\hline Centralised & 1.61 & 2.23 & 2.92 \\
\hline Decentralised & 1.45 & 2.21 & 3.00 \\
\hline \multicolumn{4}{|c|}{ Autumn - November } \\
\hline \multicolumn{4}{|l|}{ References } \\
\hline $\mathrm{RBC}$ & 15.87 & 0.37 & 11.05 \\
\hline Energy Min. & 7.65 & 0.10 & 9.44 \\
\hline \multicolumn{4}{|c|}{ DR - Static Pricing } \\
\hline Centralised & 9.35 & 0.06 & 15.78 \\
\hline Decentralised & 9.40 & 0.09 & 15.57 \\
\hline \multicolumn{4}{|c|}{ Dynamic Pricing } \\
\hline Centralised & 11.37 & 0.16 & 23.86 \\
\hline Decentralised & 11.56 & 0.21 & 23.86 \\
\hline
\end{tabular}

Figure 1 demonstrates how the call for DR changed the energy demand compared to the load-shaped de- mand profile with plots of the differences to the requested reference profiles. The dashed line represents where the level of the demand projection would have been without the load-shaping over the DR period for the decentralised MPC. Before the DR period a pre-peak was introduced in most cases as pre-heating was done to reduce consumption as much as possible during the DR period. A sustained reduction of $2 \mathrm{~kW}$ over whole DR period was not reached with the proposed MPC strategy in most cases. For example, in January and November with static pricing a considerable initial reduction was maintained for 15-20 minutes after which the demand increased above the requested reference to maintain indoor temperatures with the comfort bounds.

Overall, differences in operation between the centralised and decentralised MPC were relatively small. In March with static pricing the decentralised MPC was able to deliver a more sustained reduction compared to the centralised MPC, although the overall demand was very low over the period because demand was not high enough to deliver the full $2 \mathrm{~kW}$ reduction. In November with static pricing, the best responses could be reached, with the decentralised MPC capable of operating most of the time below the load-shaped projection. Comparisons of the demand projections made by MPC strategies showed that they were very similar prior and over the DR period in centralised and decentralised set-ups. Similarity of the solutions by decentralised and centralised MPC provided confidence that the solutions were close to optimum, although as mentioned, with the solving methodology this could not be fully guaranteed.

In all cases dynamic pricing reduced the capability to reach sustained reductions over the DR period compared to static pricing. Dynamic pricing made the MPC "pre-emptively" reduce demand over the DR period with pre-heating since the high prices coincided with it. This also set the demand projections very low, which made further reductions in demand difficult to reach, hence reducing potential for flexibility. This means that the potential revenue gained from operating under the projection would also have reduced. This was especially clear in January and November where MPC strategies reduced demand in the beginning of the DR period but were not able to maintain the reduction, significantly exceeding the reference after the initial reduction to avoid comfort violations.

\section{Effects of Prediction Inaccuracies}

To better understand MPC strategy operation, simulations were run without emulating the real building responses. This meant that dynamic disturbances and modelling errors caused by for example internal gains or heat pump operation were not accounted for. Figure 2 shows how the realised demands compared 
Differences to Requested Demand Profiles
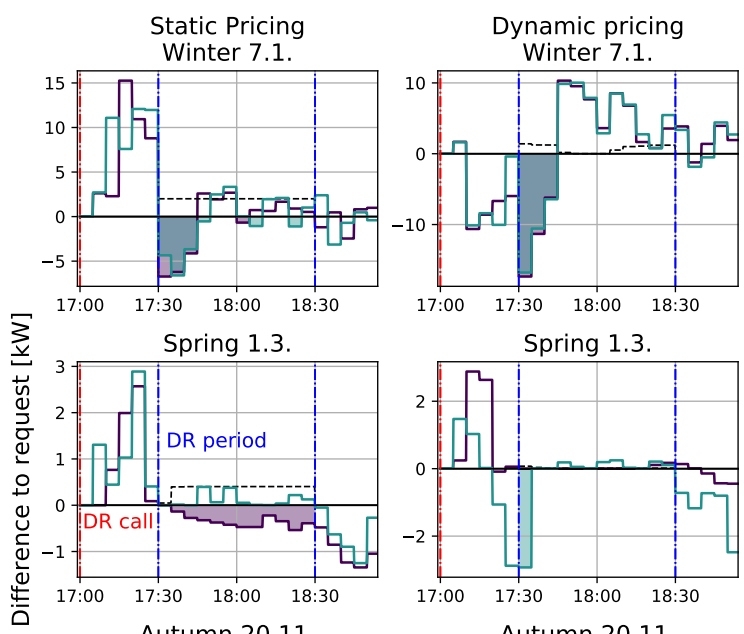

Autumn 20.11
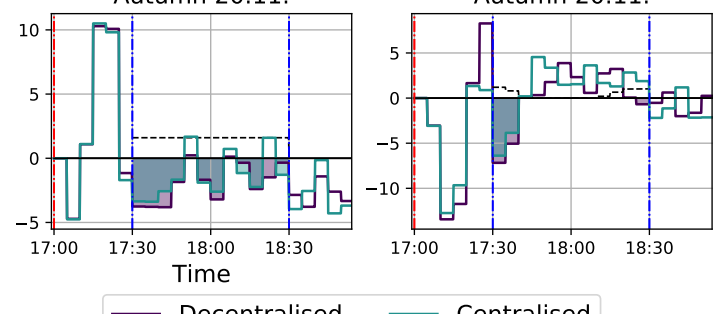

— Centralised

Figure 1: Differences between realised demand and load-shaped MPC projection during and after a call for DR. The dashed lines show the level of the reference profiles without load-shaping. Coloured areas demonstrate when reward would be received for operating below the load-shaped profile.

with the requested demand profiles in these simulations.

Again, the MPC operating with perfect predictions did not deliver a sustained reduction throughout the DR period in most cases but rather delivered a substantial initial reduction instead of a steady fixed demand reduction. In the static pricing case of January, the MPC strategies operated very similarly before the DR period but then centralised MPC was able to deliver the demand reduction, unlike the decentralised MPC. This is attributable to the centralised MPC being capable of using different buildings to allocate demand reductions most efficiently to reach the community-wide $2 \mathrm{~kW}$ reduction, unlike in the decentralised case where each building was asked for the same demand reduction regardless of their potential. This can be observed in November too with the decentralised MPC operating higher above the requested reference profile compared to the centralised MPC.

Comparing figures 1 and 2 with each other provides some useful indications of how model predictions affected flexibility. For example, in January the decentralised MPC operated for 20 minutes below the projection in the beginning of the DR period, longer than

when a real building response was emulated. Furthermore, the centralised MPC was delivered the demand reduction over the DR period when response of the real building was not considered. This highlights the importance of errors and external disturbances on MPC operation and hence the delivery of contracted flexibility in this context.

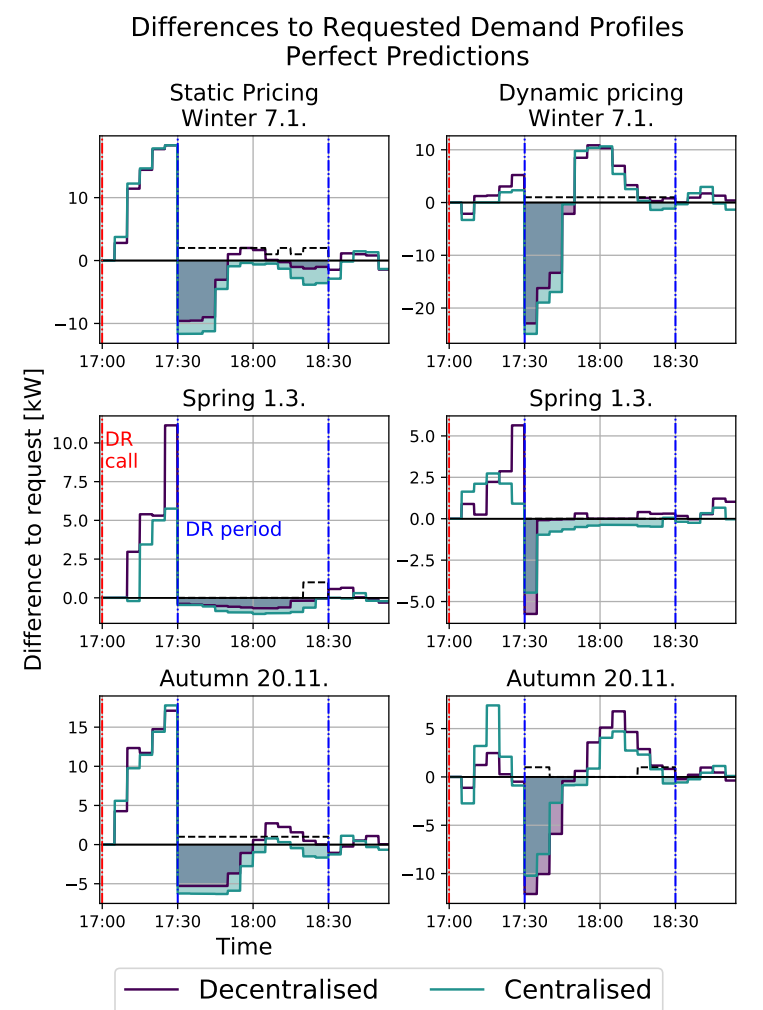

Figure 2: Differences between the realised demand and the load-shaped MPC projection during and after a call for DR when operating under perfect predictions. The dashed lines illustrate the projections without load-shaping. Coloured areas demonstrate when a reward would be received for operating below the loadshaped projection.

\section{Discussion}

\section{Factors of Flexibility}

The results overall showed how energy flexibility was affected by the pricing scheme. Dynamic pricing signals have been shown to be promising to support shifting demand. But if flexibility would be offered in real-time with dynamic pricing, making further reductions while retaining the service level would be difficult as flexibility of the system is reduced due to demand already being shifted in reaction to pricing. For an aggregator this means that potential to offer flexibility without violating comfort would be more limited by customers already responding effectively to prices.

With the proposed MPC set-up, reaching a sustained demand reduction of $2 \mathrm{~kW}$ was not provided. Typ- 
ically a significant demand reduction in the beginning of the DR period was made instead. This can be partly associated with the objective functions which incentivised and penalised equally reductions and exceedances to the requested reference profile. UK-specific building parameters were used, making the buildings thermally light-weight which might also have an effect. More insulated houses with additional thermal mass could support more sustained and longterm delivery of demand reductions and reduce the magnitude of peaks because pre-heating could also be done over longer periods of time (Masy et al., 2015). Energy efficiency measures like insulation could support enhancing flexibility potential and vice versa. This proposition and quantifying potential of such benefits in the UK-context would however still require further analysis.

An important outcome was the observed difference between the centralised and decentralised MPC strategies, demonstrating importance of the control set-up for contracted flexibility. With the centralised MPC, the load-shaped projection was set centrally on the community-level and to meet it, the MPC was free to allocate consumption within the community. In the decentralised case a fixed reduction $0.2 \mathrm{~kW}$ was requested from each house regardless of their characteristics. The centralised MPC had thus better capability to allocate demand reductions to inflict least overall costs for the community and enhance the aggregated flexibility.

\section{Limitations and Further Work}

For more general results, the methodology should be up-scaled for co-simulations of larger and more representative communities. This would also allow drawing conclusions from inspecting MPC of a community with more variability. With larger communities, more variable thermal characteristics or set-point profiles the differences between the centralised and decentralised MPC strategies might become more evident. Computational issues limited increasing the number of houses, which could be solved by improving the inefficiencies in the simulation and optimisation set up. Complexity of the emulation models could be reduced or more computation power added to run larger simulations. To ensure optimality of the optimisation solutions, the optimisation methodology should be revisited. Other methods with better guarantee of reaching an optimum solution, like linear programming, will be considered in the future.

To reduce effects of model errors on MPC operation and delivery of flexibility, the system identification methodology would also deserve more attention. Effects of initial guesses of parameters and their covariances and upper and lower bounds on model predictions were not included in the scope of the analysis. For example, tools like GreyBox could be used to perform a more elaborate system identification process
(De Coninck et al., 2016). Also data-driven models, like auto-regressive models with exogenous inputs (ARX), might provide more scalable means of developing models for control. It might be necessary to assess some of the modelling assumptions to improve predictions by the RC-models. For example, nonlinear effects of equipment like heat pumps could be included in the MPC formulation.

As further work, focus will be on exploring sensitivity of flexibility to factors like the thermal properties in buildings, set point profiles, preparation time for DR and pricing. For example, changing the ratios between the price of energy, cost for comfort violations and incentives for flexibility should change the MPC behaviour and the flexibility potential. Also, other objective function formulations will be explored to find ways of reaching more sustained reductions to cater for DR schemes. For example, formulations only penalising operation above the reference, instead of rewarding for all reductions, could be developed. Effects of uncertainties in weather predictions were left outside the scope of the paper and would need to be considered too in future implementations of the proposed MPC strategies.

\section{Conclusion}

In this study two MPC strategies, decentralised and centralised, designed for delivery of contracted flexibility by reducing demand over a set period of time based on demand projections from an economic MPC was presented. Operation of the MPC strategies were investigated with co-simulations of a community consisting of ten English homes over three case afternoons under dynamic and static pricing.

The main outcomes can be summarised as follows:

- Demand response events increased the overall energy consumption and peak power demand of the community due to pre-heating by the MPC strategies.

- Dynamic pricing reduced the flexibility potential due to pre-emptive demand shifting before the demand response period.

- Computational set-up and modelling uncertainty of the MPC strategies affected the delivery of contracted flexibility from the community.

To conclude, further analysis and work is required to develop the proposed MPC strategies for largescale implementations in real buildings. The authors are planning continue efforts aiming to find effective and scalable ways of unlocking the energy flexibility potential in communities by using simulations and experiments in test buildings.

\section{Acknowledgements}

This research was made possible by Engineering and Physical Sciences Research Council (EPSRC) sup- 
port for the London-Loughborough (LoLo) Centre for Doctoral Research in Energy Demand (grant $\mathrm{EP} / \mathrm{H} 009612 / 1)$.

\section{References}

Åkesson, J., K. E. Årzén, M. Gäfvert, T. Bergdahl, and H. Tummescheit (2010). Modeling and optimization with Optimica and JModelica.orgLanguages and tools for solving large-scale dynamic optimization problems. Computers and Chemical Engineering 34(11), 1737-1749.

Allen, E. A. and A. A. Pinney (1990). Standard dwellings for modelling: details of dimensions, construction and occupancy schedules.

BEIS (2018). Energy Consumption in the UK (ECUK) 2018.

Blum, D. and M. Wetter (2017). MPCpy: An opensource software platform for model predictive control in buildings. In IBPSA Build. Simul. Conf, Volume 2017.

CCC (2013). Fourth Carbon Budget Review.

Clauß, J., C. Finck, P. Vogler-Finck, and P. Beagon (2017). Control strategies for building energy systems to unlock demand side flexibility - A review. In IBPSA Building Simulation 2017, San Francisco, 7-9 August 2017, San Francisco. IBPSA.

Cole, W. J., D. P. Morton, and T. F. Edgar (2014). Optimal electricity rate structures for peak demand reduction using economic model predictive control. Journal of Process Control 24(8), 1311-1317.

Corbin, C. D. and G. P. Henze (2017). Predictive control of residential HVAC and its impact on the grid. Part II: simulation studies of residential HVAC as a supply following resource. Journal of Building Performance Simulation 10(4), 365-377.

De Coninck, R., F. Magnusson, J. Åkesson, and L. Helsen (2016). Toolbox for development and validation of grey-box building models for forecasting and control. Journal of Building Performance Simulation 9(3), 288-303.

Dusparic, I., A. Taylor, A. Marinescu, F. Golpayegani, and S. Clarke (2017). Residential demand response: Experimental evaluation and comparison of self-organizing techniques. Renewable and Sustainable Energy Reviews 80, 1528-1536.

El Geneidy, R. (2018). Delivery of contracted energy flexibility from communities. A Master of Research Thesis at Loughborough University.

El Geneidy, R. (2019). Github Repository of Rami El Geneidy.
El Geneidy, R. and B. Howard (2018). Review of techniques to enable community-scale demand response strategy design. uSim2018 - Urban Energy Simulation.

Haghighi, M. M. (2013). Controlling Energy-Efficient Buildings in the Context of Smart Grid : A Cyber Physical System Approach. Thesis Electrical Engineering and Computer Sciences University of California at Bekeley, 1-196.

HM Government (2016). English housing survey 2016 to 2017: Headline Report.

Jensen, S. Ø., A. Marszal-Pomianowska, R. Lollini, W. Pasut, A. Knotzer, P. Engelmann, A. Stafford, and G. Reynders (2017). IEA EBC Annex 67 Energy Flexible Buildings. Energy and Buildings 155, $25-34$.

Killian, M. and M. Kozek (2016). Ten questions concerning model predictive control for energy efficient buildings. Building and Environment 105, 403-412.

Masy, G., E. Georges, C. Verhelst, V. Lemort, and P. André (2015). Smart grid energy flexible buildings through the use of heat pumps and building thermal mass as energy storage in the belgian context. Science and Technology for the Built Environment 21(6).

Mirakhorli, A. and B. Dong (2018). Market and behavior driven predictive energy management for residential buildings. Sustainable Cities and Society 38(April), 723-735.

Ofgem (2018). State of the Energy Market 2018.

Remmen, P., M. Lauster, M. Mans, M. Fuchs, T. Osterhage, and D. Müller (2018). TEASER: an open tool for urban energy modelling of building stocks. Journal of Building Performance Simulation 11(1), 84-98.

Reynolds, J., Y. Rezgui, and J.-L. Hippolyte (2017, nov). Upscaling energy control from building to districts: Current limitations and future perspectives. Sustainable Cities and Society 35, 816-829.

Technology, E. (2012). The Effects of Cycling on Heat Pump Performance.

U S Department of Energy (2006). Benefits of Demand Response in Electricity Markets and Recommendations for Achieving Them.

Wächter, A. and L. T. Biegler (2005). On the Implementation of an Interior Point Filter Line Search Algorithm for Large-Scale Nonlinear Programming, Volume 106.

Wetter, M. and C. Van Treeck (2017). New Generation Computational Tools for Building \& Community Energy Systems Annex 60 Final Report. 